\title{
DEVELOPMENT AND TESTING OF A CAPACITIVE DIGITAL SOIL MOISTURE SENSOR WITH PRINTED CIRCUIT BOARD AS A PROBE
}

\author{
V. Ogwo ${ }^{1,}{ }^{*}$, K. N. Ogbu ${ }^{2}$, C. C. Anyadike ${ }^{3}$, O. A. Nwoke ${ }^{4}$ and C. C. Mbajiorgu 5 \\ $\mathbf{1 , 3 , 4 , 5}$, DePT. OF AGRICULTURAL \& BiORESOURCES ENGR'G, UniV. OF NigeRIA, NSUKKA. ENUGU STATE. NIGERIA \\ 2, Dept. of Agricultural \& BioresourCes EngR'G, NNAMdi AZIKIWE Univ., AWKA. ANAmbra State. NIGERIA \\ E-mail addresses: ${ }^{1}$ vintus.ogwo@unn.edu.ng, ${ }^{2}$ kn.ogbu@unizik.edu.ng, \\ ${ }^{3}$ chinenye.anyadike@unn.edu.ng, ${ }^{4}$ nwoke.oji@unn.edu.ng, ${ }^{5}$ constantine.mbarjiorgu@unn.edu.ng
}

\begin{abstract}
The quantity and quality of water present in the soil determine to a greater extent the performance of agricultural crops. Real-time determination of moisture content has a greater advantage over the traditional gravimetric method of determining soil moisture content. Thus, this work was based on the design and construction of a cost effective digital capacitive soil moisture sensor for realtime measurement. The moisture sensors comprised four integrated units namely: power supply unit with a 9V DC battery as a power source, sensor unit with a locally sourced Printed Circuit Board (PCB) as the single sensing probe, control unit made up of PIC16f877 microcontroller programmed with a $C$ language and the $C$ source code compiled in Corporate Computer Services Compiler (CSS C) compiler development environment, and a 16x2 display unit which displays the readings in percentage moisture content (\%MC) and capacitance ( $\mu F)$ of the soil obtained from the sensor on its screen. Standard gravimetric moisture content was carried out to get the calibration factor which was used to calibrate the sensor for reliability. The validation was done by taking the reprogrammed (calibrated) sensor to the field for further measurement, after which soil samples were collected for further gravimetric analysis. A regression equation was obtained by plotting the moisture content obtained from gravimetric method $\left(\% M C_{G}\right)$ against that from sensor reading $\left(\% M C_{S}\right)$ with a high degree correlation coefficient $\left(R^{2}\right)$ of 0.998 . The developed capacitive soil moisture sensor is cheap, portable, reliable and easy to use even by local farmers.
\end{abstract}

Keywords: Calibration, Capacitive sensor, Printed circuit board, Soil moisture content, Validation.

\section{INTRODUCTION}

Real-time monitoring of soil moisture and timely understanding of the soil moisture content are highly significant for crop water requirement [1-3]. Water has been described as the important requirement for agricultural production, and the right quantity and quality should be supplied to the crops at the right time so as not to over or under irrigate the crops [4]. For sustainable irrigation-based farming, water management is so important, thereby stressing the need to monitor soil water content and the use of water by plants $[5 ; 6]$. Soil moisture monitor, being the basis of the implementation of water-saving irrigation in precision agriculture, is a key link in the process to realize the automation of agricultural irrigation $[1 ; 7]$. This is because water is an essential element for the existence and sustenance of life both in plants and animals. Therefore, water must be utilized in efficient way so as to save it for future generations [8]. In order to determine and maintain optimum water required for plant growth, moisture content determination has to be carried out. The available water capacity (AWC) is the range between field capacity (FC) and permanent wilting point (PWP), which are two important water content values in soil water analysis [9]. AWC is the condition when the soil water can be absorbed by the plant roots and is widely used to assess yield potentials of crops [10]. However, determination of moisture content is important in the scheduling of irrigation and

${ }^{*}$ Corresponding author, tel: $+234-803-678-4182$ 
estimating the amount of water to apply in irrigation [5]. Therefore, direct and indirect methods are basically used in soil moisture determination. Direct method also known as gravimetric method is accurate and inexpensive, but it is time-consuming (takes a minimum of 2 days) and destructive. While, indirect methods involve the use of moisture meters in the determination of moisture content by a calibrated relationship with some other measurable variable. The suitability of these methods depends on several issues like cost, accuracy, response time, installation, management and durability. These methods are widely accepted because most of them are portable, relatively easy to operate by local farmers and give real-time values of moisture content. Capacitive sensing is one of the techniques of indirect methods that works on the principle of measuring the dielectric constant. Capacitive sensor corrects the problem of corrosion of probes of other sensing methods making them more adapted in precision agriculture [4]. The probe (sensing unit) of the developed sensor was made up of Printed Circuit Board (PCB) which acts as a capacitor in the surrounding soil-water medium. PCB uses the fringe effect to allow changes in permittivity of its surrounding medium to be identified [9]. However, the soil-water medium surrounding the probe is used as its dielectric to measure capacitance of the capacitor. A permittivity values of 80 and 5 for water and soil respectively was reported in the literature $[11 ; 9 ; 6]$, and capacitance varies directly as permittivity. Therefore, measuring the capacitance becomes a sensitive method in which to obtain the dielectric of the surrounding medium because a small change in the soil water content results to a large impact on the surrounding permittivity [9]. Detailed information on capacitive sensors can be found in $[11 ; 9 ; 4]$. PCBs are the boards that are used as the base in most electronics - both as a physical support piece and as the wiring area for the surface-mounted and socketed components. Several types of $P C B$ boards include single-layer PCBs, double-layer PCBs, multi-layer PCBs, rigid PCBs, flexible PCBs, rigid-Flex PCBs, high-frequency PCBs, and aluminum-backed PCBs. This study encompasses development and testing of digital soil moisture sensor using discarded printed circuit board (PCB) as a sensing probe to determine the soil moisture. The developed sensor is a simple, compact, portable and auto ranging electronic device which measures soil moisture content accurately and precisely. By knowing the moisture value, the farmer can estimate when and how much to water the fields so as to avoid overwatering or wilting of crops [5]. These practices will increase crop yield, improve quality of crops, conserve water resources, save energy, and decrease fertilizer supplies. Most researchers have made efforts into the development of different types of devices for measurement of soil moisture for agricultural purposes in Nigeria. Onoja et al., [5] developed a resistive soil moisture meter using the 555 integrated circuit timer which indicates the resistance of the soil under study thereby measuring the moisture content of the soil. The probe of resistive sensor is prone to corrosion which greatly affects its readings, and this limitation can be corrected by capacitive sensing method. Ogbu et al., [4] developed a capacitive metre using the NE555 timer circuit. Ogbu et al.'s meter is made up of two probe wires as the sensor which measure the soil moisture capacitance corresponding to a definite frequency that is always directly proportional to the soil moisture content. Ogbu et al.'s meter is affected by the capacitance of the air which is greater than that of moisture in the soil. This limitation was improved upon in the present study by incorporating the permittivity of air in the design. Therefore, the objectives of this study are to: develop a low-cost soil moisture sensor; calibrate the moisture sensor with data and calculations obtained from the gravimetric method of moisture content $\left(\mathrm{MC}_{\mathrm{G}}\right)$ measurement; and validate the instrument.

\section{MATERIALS AND METHODS}

\subsection{Circuit Units Design and Construction}

The materials used in the development of the digital sensor are classified into power supply unit, sensing unit, control unit and display unit. Fig. 1 is a complete circuit diagram of the developed moisture sensor. The power supply unit is made up of a 9V DC battery, battery clip, $10 \mu \mathrm{F}$ by $25 \mathrm{~V}$ electrolytic capacitor, $5 \mathrm{~V}$ voltage regulator (7805), a switch, a red-light emitting diode (LED - as indicator) and a $1 \mathrm{k} \Omega$ resistor. The $9 \mathrm{~V}$ DC battery produces $9 \mathrm{~V}$ direct current supply, which the 7805-voltage regulator regulates to steady $5 \mathrm{~V} D C$. This is because the microcontroller (pic16f877A), the liquid crystal displays (LCD) and the 555 timer IC require 5 volts direct current supply. The switch was used to make and break the circuit in order to conserve the battery's energy. The red LED was used as an indicator, to indicate when the device is on. The 1 kilo ohm resistor was used to limit current and voltage for 
the LED since the LED requires 2-3V DC supply. The $10 \mu \mathrm{F}$ by $25 \mathrm{~V}$ electrolytic capacitor was used to filter off ripples from the regulator's output. The sensing unit is made up of a locally sourced PCB capacitive sensor (obtained from a damaged rechargeable lantern), a 555 timer IC (configured in astable mode), two 100 ohms resistors, and $0.01 \mu \mathrm{F}$ capacitor. The 555 timer IC, when configured in astable mode, oscillates and produces clock pulses in its output pin. According to $[5 ; 13]$, the frequency, $f$ of the oscillation is affected by the values of resistance $R 1$, resistance $\mathrm{R} 2$ and capacitance $\mathrm{C}$ as in Eqn. (1):

$$
f=\frac{1}{0.693(R 1+2 R 2) C}
$$

where, R1 is the resistance of the resistor between the Vcc (pin 8) and the discharge pin (pin7) of the 555 timer, R2 is the resistance of the resistor between the discharge pin the threshold pin (pin 6) of the IC, $C$ is the capacitance of the sensor in Farad existing between pin 6 and GND (ground) of the IC and $f$ is the frequency (in Hertz).

Then, the capacitance of the sensor, $\mathrm{C}$ can be estimated from Eqn. (1) as:

$$
C=\frac{1}{0.693(R 1+2 R 2) f}
$$

However, the frequency of oscillation of the 555 timer IC ( $f$ ) is needed to measure the capacitance of the sensor. Thus, to determine this frequency, the output pin of the IC (pin 3) was connected to timer of the microcontroller, which was programmed to count the number of clock pulses received in one second (i.e. number of cycles per second which is the required frequency). The signal from the sensor is amplified within the 555 timer IC by an NPN bipolar junction transistor configured in common emitter mode for signal voltage amplification.

Also, capacitance of the sensor in free space was estimated by:

$$
C=\frac{\left(\varepsilon \varepsilon_{0} A\right)}{d}
$$

where $d$ is the distance between the plates $(0.001 \mathrm{~m})$, $\varepsilon$ is the relative electric permittivity of the dielectric material and is 80 for water, $\varepsilon_{0}$ is the permittivity of space and is taken as 1 for air [11], and $A$ is the area of the capacitive sensor plate estimated as $0.0058 \mathrm{~m}^{2}$ using Eqn. (4):

$$
A=2\left\{\frac{1}{2}(a+b) h\right\}+(L \times W)
$$

where $a$ is the shorter length of trapezium that made up the distal end of the capacitive sensor plate $(0.025 \mathrm{~m}), \mathrm{b}$ is the longer length of the trapezium that made up one of the distal ends of the sensor plate $(0.052 \mathrm{~m}), \mathrm{h}$ is the height of the trapezium $(0.024 \mathrm{~m})$, $L$ is the length of the rectangular part of the capacitive plate $(0.076 \mathrm{~m}), \mathrm{W}$ is the width of the rectangular part of the sensor plate $(0.052) \mathrm{m}$. Thus, substituting the above parameters into Eqn. (3), $C$ in free space was calculated as $469.8 \mathrm{~F}$. Then, the moisture content of the soil in percentage (\%MCs) as displayed on the LCD screen is the ratio of the capacitance of the sensor in the presence of water to the capacitance of the sensor in free space as given in Eqn. (5):

$$
\% M C_{S}=\frac{C}{469.8} \times 100
$$

where $\mathrm{C}$ is the capacitance of the sensor in the presence of water.

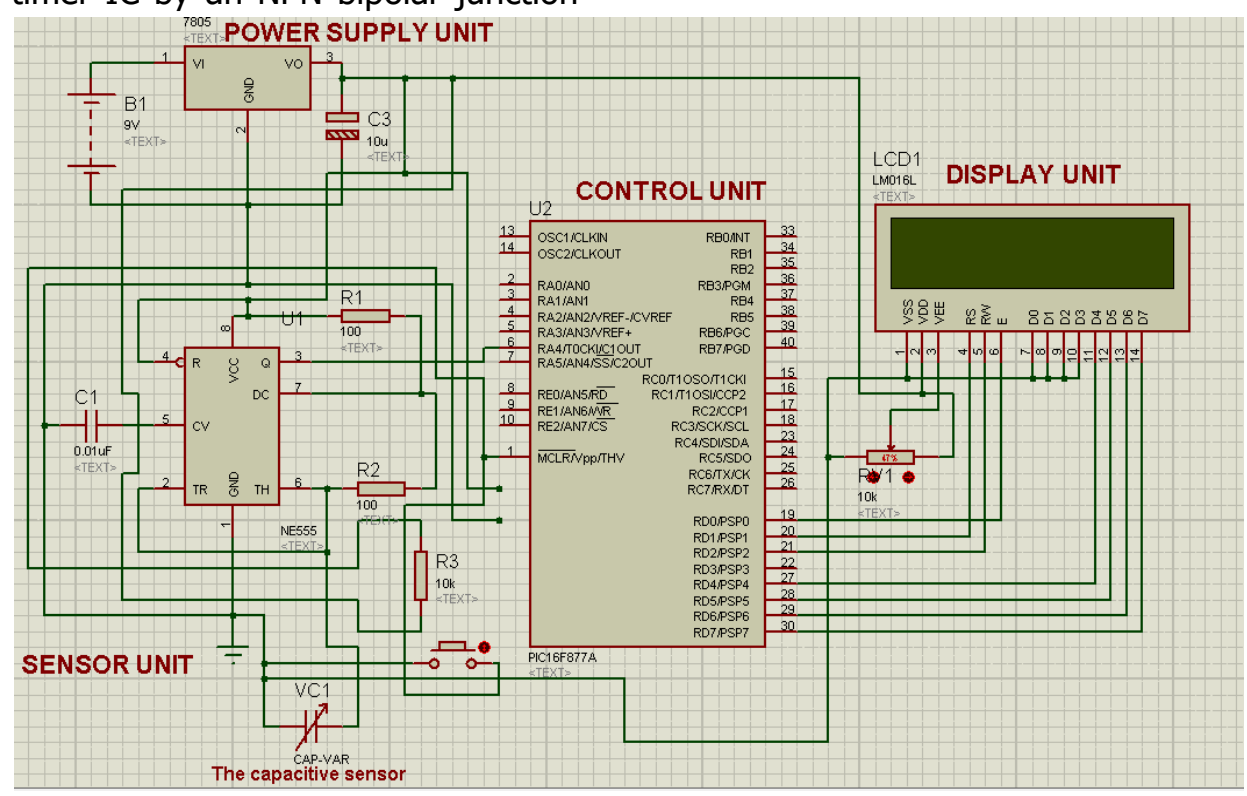

Fig. 1: Circuit diagram consisting of the categorized units 
The control unit is made of Programmable Interface Controller 16f877A (PIC16f877A) microcontroller. This was programmed with $C$ language. The $C$ source code was compiled in Corporate Computer Services Compiler (CCS C) development environment. The hexadecimal file of the program was written to the microcontroller using kit 2 Programmable Interface Controller (PIC kit 2) programmer. The display unit is made up of 16 by 2 Liquid Crystal Display (LCD). Its job is to display the readings obtained from the sensor on its screen. Fig. 2 is the photograph of the developed, assembled and packaged circuit of the digital soil moisture sensor in Fig. 1.

\subsection{Calibration of the Capacitive Digital Soil Moisture Sensor}

A typical Sandy Clay Loam soil sample was used for the calibration of this digital soil moisture sensor. Thirteen (13) weighed moisture cans, soil auger and the developed capacitive soil moisture sensor device were taken to the field for soil samples collection. Soil auger was used to excavate soil samples at a depth of $30 \mathrm{~cm}$ at various locations with varying moisture contents, and the capacitance of the excavated sample taken with the developed capacitive soil moisture sensor device. Thereafter, soil at the locations was equally collected with moisture cans and taken to the laboratory for gravimetric percentage moisture content $\left(\% \mathrm{MC}_{\mathrm{G}}\right)$ determination. Eqn. (6) was used to determine the (\%MC $)_{\mathrm{G}}$ ) after the samples were weighed and oven dried at $105{ }^{\circ} \mathrm{C}$ for 24 hours and reweighed. A calibration factor, which when multiplied with the capacitance gives the gravimetric moisture content was calculated for each sample (Table 1). This was obtained by dividing the (\%MC $\left.{ }_{\mathrm{G}}\right)$ of the sample by the respective capacitance of the samples measured with the developed moisture sensor device as given in Eqn. (7). The average of these calculated calibration factors was used for the reprogramming of the developed sensor for reliability. A graph of gravimetric moisture content $\left(\% \mathrm{MC}_{\mathrm{G}}\right)$ versus capacitance known as calibration curve was used to calibrate the developed capacitive soil moisture sensor.

$$
\% M C_{G}=\frac{W_{w e t}-W_{d r y}}{W_{\text {wet }}} \times 100
$$

where $\% \mathrm{MC}_{\mathrm{G}}$ is the gravimetric moisture content of soil sample in percentage (wet basis), $\mathrm{W}_{\text {wet }}$ is the weight of moist soil sample and $\mathrm{W}_{\mathrm{dry}}$ is the weight of oven-dried soil sample.

$$
\text { Calibration factor }=\frac{\% M C}{\text { Capacitance }}
$$

\subsection{Validation of the Capacitive Soil Moisture Sensor}

In order to validate the capacitive soil moisture sensor, the sensor device was taken to the field and used to measure soil moisture contents ( $\left.\% M C_{S}\right)$. The field measurements were made at random places with varying moisture contents after which soil samples at the points where the readings were taken were collected and taken to the laboratory for gravimetric soil moisture analysis to determine the $\% \mathrm{MC}_{\mathrm{G}}$. The results of moisture content obtained from gravimetric analysis $\left(\% \mathrm{MC}_{\mathrm{G}}\right)$ and the device moisture content readings (\%MCs) were compared to know the reliability of the sensor.

\section{RESULTS AND ANALYSIS}

The capacitive digital soil moisture sensor produced with a cheap improvised PCB as the probe is shown in Fig. 2 . The readings of capacitance $(\mu \mathrm{F})$ and percentage moisture content (\%MC) are displayed on the LCD.

\subsection{Calibration}

The need to calibrate a developed instrument against a standard is a necessary link in sensor design process $[1 ; 12]$. The results that were used for the calibration of the capacitive soil moisture sensor as obtained from the laboratory gravimetric method is shown in Table 1. The average calibration factor was obtained using Eqn. (7) as 219.4249. This was used to reprogram the moisture meter. For every capacitance value of the soil detected by the sensor is multiplied by the average calibration factor to give the percentage moisture content which is displayed on the screen. Fig. 3 shows the calibration curve of gravimetric moisture content $\left(\% \mathrm{MC}_{\mathrm{G}}\right)$ against sensor capacitance reading $(\mu \mathrm{F})$ with the correlation coefficient $\left(R^{2}\right)$ value of 0.98 . These calibration curve and calibration factor were used for further reprogramming of the sensor microcontroller during calibration process to ensure reliability of the sensor. 


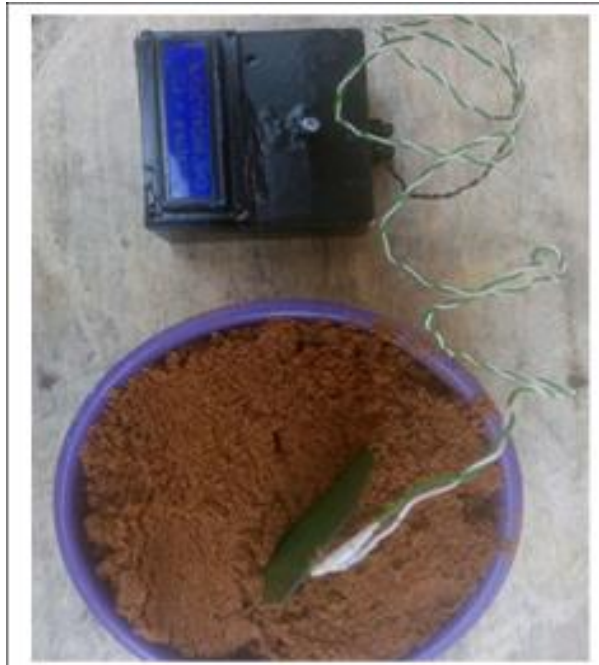

(a)

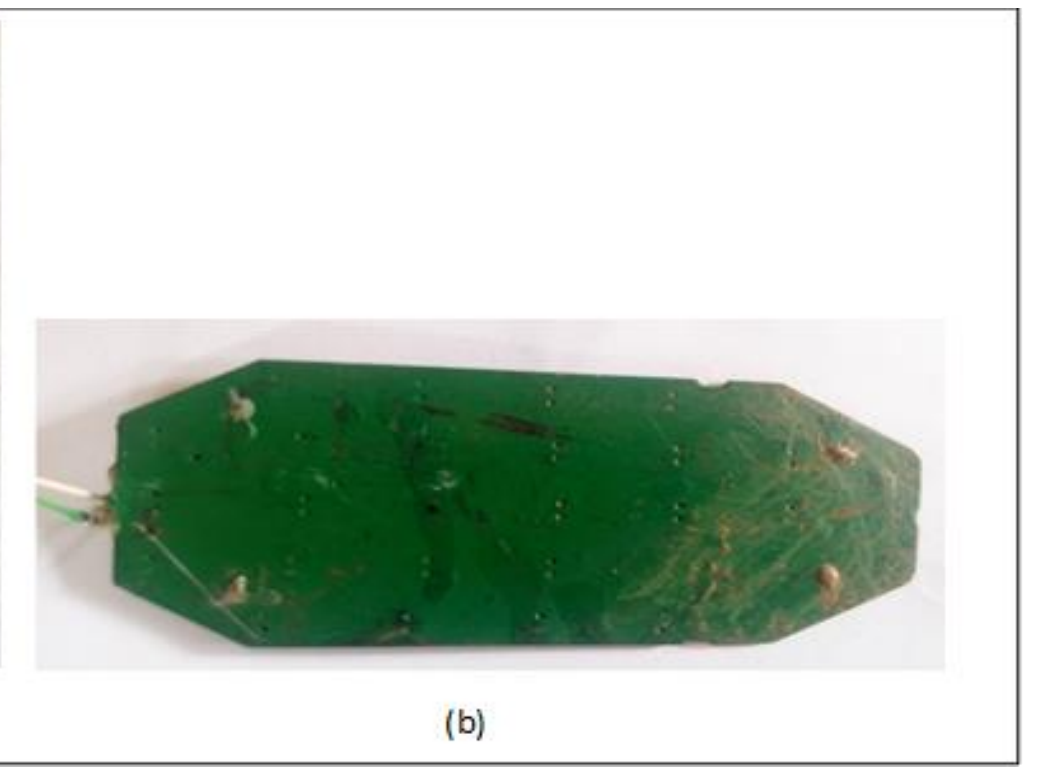

(b)

Fig. 2: (a) Soil moisture meter being used to measure percentage moisture content (b) Sensor Probe

Table 1: Soil Moisture Sensor Capacitance Reading and Gravimetric Moisture Content (\%)

\begin{tabular}{cccccc}
\hline Wwet $(\mathrm{g})$ & Wdry $(\mathrm{g})$ & Wwater $(\mathrm{g})$ & $\%_{\mathrm{MC}}$ & Sensor Capacitance Reading $(\mu \mathrm{F})$ & Calibration factor \\
\hline 0 & 0 & 0 & 0 & 0 & 0 \\
97.8 & 88.0 & 9.8 & 10.02045 & 0.041 & 244.4012 \\
105.9 & 95.1 & 10.8 & 10.1983 & 0.044 & 231.7796 \\
105.6 & 94.5 & 11.1 & 10.51136 & 0.048 & 218.9867 \\
96.4 & 85.7 & 10.7 & 11.09959 & 0.051 & 217.6389 \\
134.9 & 119.7 & 15.2 & 11.26761 & 0.057 & 197.6773 \\
109.7 & 96.9 & 12.8 & 11.66819 & 0.061 & 191.2817 \\
134.2 & 114.9 & 19.3 & 14.38152 & 0.064 & 224.7113 \\
125.6 & 107.0 & 18.6 & 14.80892 & 0.066 & 224.3775 \\
95.5 & 80.7 & 14.8 & 15.49738 & 0.070 & 221.3912 \\
100.8 & 84.5 & 16.3 & 16.17063 & 0.074 & 218.5221 \\
110.5 & 91.7 & 18.8 & 17.01357 & 0.077 & 220.9555 \\
120.2 & 98.9 & 21.3 & 17.72047 & 0.081 & 218.7712 \\
115.1 & 92.1 & 23.0 & 19.98262 & 0.090 & 222.0292 \\
\hline Average & & & & 219.4249
\end{tabular}

Where: Wwet is weight of wet soil, Wdry is weight of oven dry soil, Wwater is weight of water, $\%_{\mathrm{MC}} \mathrm{G}_{\mathrm{G}}$ the gravimetric percentage moisture content.

\subsection{Validation}

The calibrated digital moisture sensor was used to measure soil moisture contents at randomly selected eleven (11) locations with varying moisture contents with the aid of the calibration curve earlier obtained. The moisture content (\%) and capacitance $(\mu \mathrm{F})$ at these various locations were read off from the LCD of the developed sensor. Also, soil samples at these locations were taken for further gravimetric analysis. The results of these measurements and the gravimetric moisture content for the soil samples from the respective locations are shown in Table 2. The comparison of the results between the moisture contents obtained from the sensor reading and that obtained from gravimetric method showed a very high correlation of $\mathrm{R}^{2}$ of 0.998 as shown in Fig. 4.

\subsection{Discussion}

During calibration process, the sensor capacitance reading for every location randomly selected and the gravimetric $\% \mathrm{MC}_{\mathrm{G}}$ for the soil samples of the same locations were as shown in Table 1. 


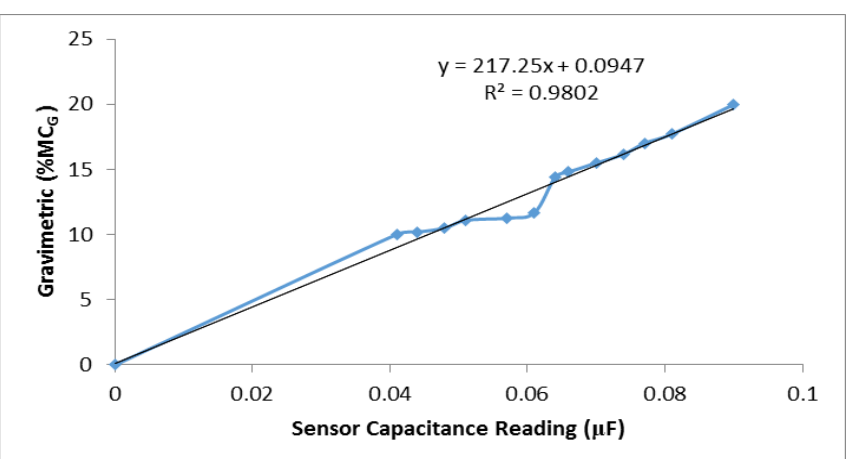

Fig. 3: Calibration Curve for Developed Soil Moisture

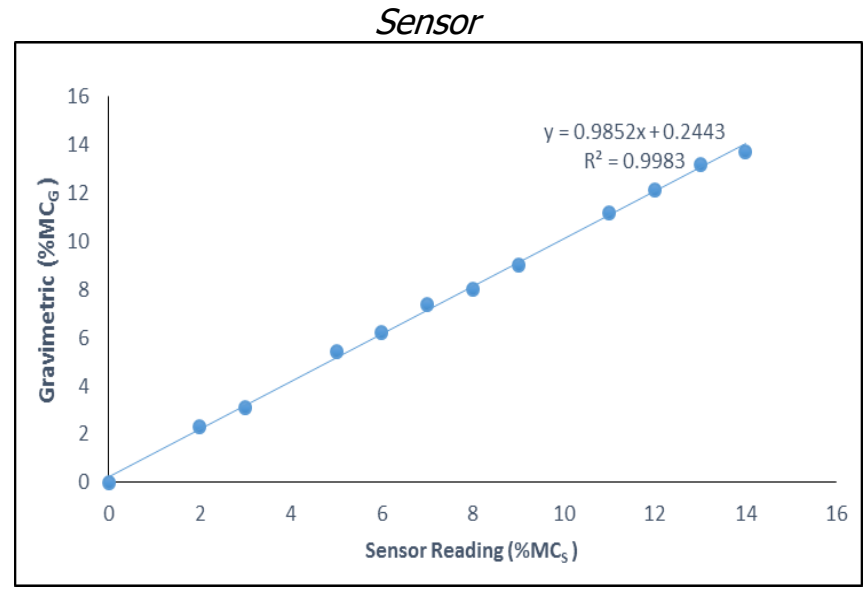

Fig. 4: Comparison Between Moisture Content from Sensor Reading $\left(\% M C_{S}\right)$ and Gravimetric moisture content $\left(\% M C_{G}\right)$

Table 2: Capacitance and moisture content reading from meter and gravimetric method.

\begin{tabular}{cccc}
\hline $\begin{array}{c}\text { Sensor } \\
\text { Reading } \\
\mathrm{MC}_{S}(\%)\end{array}$ & $\begin{array}{c}\text { Gravimetric } \\
\mathrm{MC}_{\mathrm{G}}(\%)\end{array}$ & $\begin{array}{c}\text { Difference } \\
(\%)\end{array}$ & $\begin{array}{c}\text { Capacitance } \\
(\mu \mathrm{F})\end{array}$ \\
\hline 0.00 & 0.00 & 0.00 & 0.00 \\
2.0 & 2.3 & 0.30 & 0.012 \\
3.0 & 3.1 & 0.10 & 0.014 \\
5.0 & 5.4 & 0.40 & 0.026 \\
6.0 & 6.2 & 0.20 & 0.030 \\
7.0 & 7.4 & 0.40 & 0.035 \\
8.0 & 8.0 & 0.00 & 0.038 \\
9.0 & 9.0 & 0.00 & 0.041 \\
11.0 & 11.2 & 0.20 & 0.052 \\
12.0 & 12.1 & 0.10 & 0.056 \\
13.0 & 13.2 & 0.20 & 0.060 \\
14.0 & 13.7 & 0.30 & 0.063 \\
\hline \multicolumn{4}{c}{ Mean deviation } \\
\hline
\end{tabular}

The average calibration factor of 219.4249 obtained from Eqn. (7) was used to reprogram the sensor by coding it to multiply every capacitance value of the soil detected by this factor to give the \%MCs which would be displayed on the LCD screen. It was observed from Table 1 that there was a slight increase in the capacitance of the soil samples as the gravimetric $\%_{M C_{G}}$ increases from one sample to another. Calibration curve of gravimetric $\% \mathrm{MC}_{\mathrm{G}}$ against sensor capacitance reading ( $\mu \mathrm{F}$ ) (Fig. 3) showed a high correlation coefficient $\left(R^{2}\right)$ value of 0.98 which is an indication of a high degree of sensor reliability to measure \%MC. This high correlation coefficient $\left(R^{2}\right)$ value was comparable to the capacitive metre developed by Ogbu, et al., [4] with $R^{2}$ value of 0.984 during calibration process. It was observed from Table 2 that the difference in the measurements of moisture contents obtained between gravimetric method and the calibrated sensor reading ranged from $0.0 \%$ to $0.4 \%$ with a mean deviation of $0.145 \%$. The soil moisture content determined with this sensor is more reliable than the resistive sensor developed by Onoja, et al., [5] which has the mean deviation of $0.512 \%$ from gravimetric moisture content. This could be attributed to the fact that Onoja, et al.'s soil moisture meter is of resistive types that relate soil moisture content to the resistance of the soil under study. The calibrated sensor meter was validated by comparing between the moisture contents (\%MC) obtained from the sensor reading and that obtained from gravimetric method. The correlation coefficient $\left(R^{2}\right)$ value of 0.9983 was observed indicating that the developed sensor meter could provide up to $99.8 \%$ accuracy in measuring the percentage soil moisture content. The accuracy of this developed sensor is higher when compared with Ogbu et al., [4] developed soil moisture meter with the regression value $\left(R^{2}\right)$ of 0.964 during validation. It is, therefore, recommended to carry out calibrations of different soil types since the design of the sensor assumed uniformity in the soil types. Also, further study should be carried out to investigate the response of the sensor on changes in soil salinity.

\section{CONCLUSION}

The digital capacitive soil moisture sensor designed is very simple to understand, handle and can be operated by all age-groups of farmers. It can be used to check the moisture value for any crop. Sensor can be placed vertically in the soil to check the depth of irrigated water and also it can be placed horizontally at different heights in the soil according to the crop. It is user friendly and can also be used by uneducated farmers. The device works on the principle of capacitance and can be reprogrammed to add more features with the microcontroller. The choice of capacitive probe is to avoid the deterioration of the 
probe by corroding thereby making it to have a longer functional life. This was achieved by coating the probe with insulator. The Light Crystal Display (LCD) gives a capacitive output corresponding to the conductivity of the soil. The device was programmed to convert the capacitance of the soil into percentage moisture content (\%MC) which displays on the LCD screen. The capacitance of the soil varies depending upon the amount of moisture present in it. The meter was calibrated and validated to obtain a regression coefficient $\left(R^{2}\right)$ of 0.998 that shows a reliable variation of capacitance with respect to the moisture content. The cost of the construction is relatively cheap. The meter has high precision and can provide up to $99.8 \%$ accuracy in estimating the value of the soil moisture content.

\section{REFERENCES}

[1] Zhen, X., Changjun, S., Hua, Y. and Shirui, Z. Research and design of soil water content sensor based on high-frequency capacity. Sensors \& Transducers, Vol. 26, Special Issue, March 2014, pp. 56-60.

[2] Wang Xiaolei, Hu Jiandong, Jiang Min. et al. Experiment on fast-measurement of soil moisture based on additional resistance method, Transactions of the CSAE, 2009, 25, 10, pp. 7681.

[3] Bell J. P., Dean T. J. and Baty A. J. B. Soil moisture measurement by an improved capacitance technique, Part II: field techniques, evaluation and calibration, Journal of Hydrology, 93, 1, 1987, pp. 79-90.

[4] Ogbu, K.N., E.L. Ndulue, Ogwo, V. and Mbajiorgu, C.C. Development and testing of a capacitive digital soil moisture metre, Nigerian Journal of Technology (NIJOTECH), Vol. 35, No. 3, July 2016, pp. $686-693$
[5] Onoja, S.B., Enokela, J.A. and Ebute, G.O. A Digital soil moisture meter using the 555 timer. ARPN Journal of Engineering and Applied Sciences, VOL. 9, NO. 10, October 2014, pp. 19941998.

[6] Evett, S.R. (2007). Soil water and monitoring technology. In: R.J. Lascano and R.E. Sojka (Ed.). Irrigation of Agricultural crops. Agron. Monogr. 30, $2^{\text {nd }}$ Ed. ASA, CSSA, and SSSA, Madison, WI, 2007, pp. 25-84.

[7] Luo Xiwen, Zang Ying, Zhou Zhiyan, Research progress in farming information acquisition technique for precision agriculture, Transactions of the CSAE, 2006, 22, 1, pp. 67-173.

[8] Khanna, N., Singh, G., Jain, D.K. and Kaur, M. (2014). Design and Development of Soil Moisture Sensor and Response Monitoring System. International Journal of Latest Research in Science and Technology, Volume 3, Issue 6: Page No.142145

[9] Harris, N.R. and Stonard, A. A printed capacitance sensor for soil moisture measurement, Proceedings, December3, 2018, 2, 705

[10] Robinson, D.A.; Campbell, C.S.; Hopmans, J.W.; Hornbuckle, B.K.; Jones, S.B.; Knight, R.; Ogden, F.; Selker, J.; Wendroth, O. Soil Moisture Measurement for Ecological and Hydrological Watershed-Scale Observatories: A Review. Vadose Zone J. 2007, 7, 358-389.

[11] Terzic, E., Terzic, J., Nagarajah, R. and Alamgir, M. A neural network approach to fluid quantity measurement in dynamic environments. Copy right: Springer-Verlag, London, 2012, p. 15.

[12] Whalley W. R., Cope R. E., Nicholl C. J. and Whitmore, A.P. Infield calibration of a dielectric soil moisture meter designed for use in an access tube. Soil Use and Management, 20, 2004, pp. 203-206.

[13] Millman J. and Grabel A. Microelectronics. McGraw-Hill Book Company, New York, USA. 1988 